ARTICLE

\title{
ptk7 mutant zebrafish models of congenital and idiopathic scoliosis implicate dysregulated Wnt signalling in disease
}

\author{
Madeline Hayes ${ }^{1,2}$, Xiaochong Gao ${ }^{3}$, Lisa X. Yu ${ }^{4}$, Nandina Paria ${ }^{3}$, R. Mark Henkelman ${ }^{4,5}$, Carol A. Wise ${ }^{3,6}$ \\ \& Brian Ciruna ${ }^{1,2}$
}

Scoliosis is a complex genetic disorder of the musculoskeletal system, characterized by threedimensional rotation of the spine. Curvatures caused by malformed vertebrae (congenital scoliosis (CS)) are apparent at birth. Spinal curvatures with no underlying vertebral abnormality (idiopathic scoliosis (IS)) most commonly manifest during adolescence. The genetic and biological mechanisms responsible for IS remain poorly understood due largely to limited experimental models. Here we describe zygotic ptk7 (Zptk7) mutant zebrafish, deficient in a critical regulator of Wnt signalling, as the first genetically defined developmental model of IS. We identify a novel sequence variant within a single IS patient that disrupts PTK7 function, consistent with a role for dysregulated Wnt activity in disease pathogenesis. Furthermore, we demonstrate that embryonic loss-of-gene function in maternal-zygotic ptk7 mutants (MZptk7) leads to vertebral anomalies associated with CS. Our data suggest novel molecular origins of, and genetic links between, congenital and idiopathic forms of disease.

\footnotetext{
${ }^{1}$ Program in Developmental \& Stem Cell Biology, The Hospital for Sick Children, 686 Bay Street, PGCRL 15-9712, Toronto, Ontario, Canada M5G 0A4. ${ }^{2}$ Department of Molecular Genetics, The University of Toronto, Toronto, Ontario, Canada M5S 1A8. ${ }^{3}$ Sarah M. and Charles E. Seay Center for Musculoskeletal Research, Texas Scottish Rite Hospital for Children, 2222 Welborn Street, Dallas, Texas 75219, USA. ${ }^{4}$ Mouse Imaging Centre (MICe), The Hospital for Sick Children, 25 Orde Street, Toronto, Ontario, Canada M5T 3H7. ${ }^{5}$ Department of Medical Biophysics, The University of Toronto, Toronto, Ontario, Canada M5G 2M9. ${ }^{6}$ Departments of Orthopedics, Pediatrics, McDermott Center for Human Growth and Development, University of Texas Southwestern Medical Center at Dallas, Dallas, Texas 75253, USA. Correspondence and requests for materials should be addressed to B.C. (email: ciruna@sickkids.ca).
} 
S coliosis refers to spinal curvature $>10$ degrees and can occur as a consequence of vertebral malformations, underlying congenital or juvenile disease, or with no identifiable primary cause ${ }^{1,2}$. Scoliosis caused by congenital vertebral malformation (CVM) is referred to as congenital scoliosis (CS) and affects $0.13-0.5$ of 1,000 live human births ${ }^{1,3}$. CVMs like hemivertebrae, wedge-shaped vertebrae, vertebral fusions and/or vertebral bar can exist throughout the spine and often impose severe deformity on affected individuals. In mammals, vertebral bodies are derived from somites-periodic segments of paraxial mesoderm that form in pairs along the cranial-to-caudal axis in response to an internal 'segmentation clock'. Briefly, a molecular network of Notch, canonical Wnt/ $\beta$-catenin and FGF signalling pathways generate travelling waves of gene expression along the posterior body axis that drive rhythmic somite production from the presomitic mesoderm (PSM) (reviewed in ref. 4). To date, most mutations associated with human CS disrupt Notch segmentation genes ${ }^{5-9}$. However, the genetic basis of the majority of CVMs remains unknown.

The most prevalent forms of human spinal curvature are idiopathic (that is, with no underlying pathology). Idiopathic scoliosis (IS) affects $\sim 3 \%$ of children worldwide, with many individuals eventually requiring some form of clinical intervention $^{1,10}$. IS typically presents during adolescence as a threedimensional rotation of the spine that has a propensity to increase in magnitude up to sexual maturity. These deformities range in both severity and progression with a significant bias for severe curves in females ${ }^{11,12}$. Familial clustering and concordance among monozygotic twins indicates a primary genetic cause for IS (refs 13,14) and genome-wide association studies have identified polymorphisms associated with IS in divergent human populations ${ }^{15-17}$. However, phenotypic and genetic heterogeneity have typically made it difficult to define causative mutations in humans.

Limitations inherent to the available developmental and genetic animal models of scoliosis have slowed our basic understanding of IS pathogenesis ${ }^{18,19}$. Fundamentally, scoliosis does not readily develop in most laboratory animals and induction of IS-like deformities requires drastic surgical or systemic interventions that preclude relevance to human disease $^{18}$. Rodents are quadrupeds and the structure of their spine and centre of gravity are quite different from humans. Tellingly, rodents only become susceptible to spinal curves when forced to walk upright ${ }^{20,21}$, revealing the importance of biomechanics and spinal loading during IS progression. Bipedalism has made induced chick models popular; however, in chick, anatomical restraints on spinal mobility are likely protective and prevent the full range of spinal curvatures that can develop in humans ${ }^{22}$.

In contrast, spinal deformity is naturally common among teleosts, with nutritional, environmental and/or genetic factors associated with curvature in different species and fish populations ${ }^{12,23}$. Teleosts experience cranial-to-caudal spinal loads (similar to humans) that are generated as a function of swimming forward through dense media/water coupled with caudal tail-propulsion. Thus, similar biomechanical forces along the spine may make fish more susceptible to late-onset spinal curvatures $^{12}$. Interestingly, heritable scoliosis-like phenotypes have been characterized in fish raising the intriguing possibility that teleosts can effectively model human IS $^{12,23}$. However, the genetic lesions responsible for late-onset spinal curvatures in these fish models have not yet been identified.

We have previously reported the generation of protein tyrosine kinase 7 ( $p t k 7$ ) mutant zebrafish ( $p t k 7 a$, Ensembl Zv9; ref. 24). Ptk7 is an evolutionarily conserved atypical receptor tyrosine kinase that has been implicated in vertebrate Wnt,
Semaphorin/Plexin and VEGF signal transduction ${ }^{24-30}$. Our analysis of patterning and morphogenesis defects in maternalzygotic ptk7 mutant embryos (MZptk7), which demonstrate early and complete loss-of-gene function, revealed essential roles for Ptk7 in regulating multiple branches of Wnt signal transduction $^{24}$. Specifically, we demonstrated a key role for Ptk7 in potentiating non-canonical Wnt/planar cell polarity (PCP) signals that coordinate polarized cell movements to shape the body axis during gastrulation and neurulation ${ }^{31}$, as well as negatively regulating canonical $\mathrm{Wnt} / \beta$-catenin activity ${ }^{24}$. Remarkably, embryonic defects are fully rescued in Zptk7 mutant embryos, highlighting an important role for early maternal gene function in developmental patterning and morphogenesis.

In this study we characterize Zptk7 and MZptk7 mutant zebrafish as functional models of idiopathic and congenital scoliosis, respectively. We demonstrate that zygotic $p t k 7$ mutant fish display fully penetrant late-onset spinal curvature that is not associated with vertebral malformations, is sexually dimorphic and variable in both time of onset and severity. Our work thus establishes Zptk7 mutant zebrafish as the first genetically defined developmental model of human IS. Moreover, we demonstrate that a novel PTK7 mutation identified in a single human IS patient disrupts both $\mathrm{PCP}$ and $\mathrm{Wnt} / \beta$-catenin signalling, further suggesting that dysregulated Wnt activity may contribute to disease. Conversely, early loss of Ptk7 function in MZptk7 mutant larvae results in CS-like vertebral malformations. These CVMs may be attributed to earlier defects in segmentation caused by PCP and Wnt/ $\beta$-catenin signalling defects. Together, our data suggests a genetic link between CS and IS and highlights the utility of zebrafish for modelling human spinal deformities.

\section{Results}

Zptk7 mutants develop late-onset spinal curves that model IS. Zptk7 mutant embryos do not show defects in embryonic patterning or morphogenesis, as maternal Ptk7 is sufficient to rescue early development ${ }^{24}$. However, at late larval and early juvenile stages, all Zptk7 mutant fish develop scoliosis with spinal curvatures of variable magnitude observed in both the sagittal and coronal axes (Fig. 1a,b). In zebrafish, spinal deformities can often be caused by Pseudolomoa neurophilia, Aeromonas hydrophila, and mycobacterium spp. infection. To determine whether observed scoliosis was secondary to an infectious agent, Zptk7 mutant fish with severe spinal curvatures were sent to the Zebrafish International Resource Center Health Services (Eugene, Oregon) for histopathology. All samples tested negative for infection, indicating a more direct link between $p t k 7$ mutation and spinal curve development.

To determine whether observed scoliosis resulted from CVMs, vertebrae formation was imaged in Zptk7 mutant and control animals using calcein stain (a vital, fluorescent calciumbinding chromophore) ${ }^{32}$. Larvae were analyzed at $4,4.5$ and $6.0 \mathrm{~mm}$ standard length. No defects in vertebral patterning or formation were observed in Zptk7 mutants $(n=33)$ compared with $p t k 7 /+$ siblings $(n=40$; Fig. $2 \mathrm{a}-\mathrm{f})$. High-resolution micronscale computed tomography (microCT) was then performed to further examine spinal curvature and vertebral structure in Zptk7 mutant zebrafish. Three-dimensional skeletal reconstructions of 12 Zptk7 mutant adults and 3 ptk $7 /+$ siblings revealed the profound late-onset curvatures typical of mutant fish and confirmed that abnormal vertebral patterning is not a general aspect of the Zptk7 mutant phenotype (Fig. 1i,m; Supplementary Movies 1-5).

To characterize vertebral shape changes, we measured the length of individual thoracic and tail vertebrae along the dorsal, 

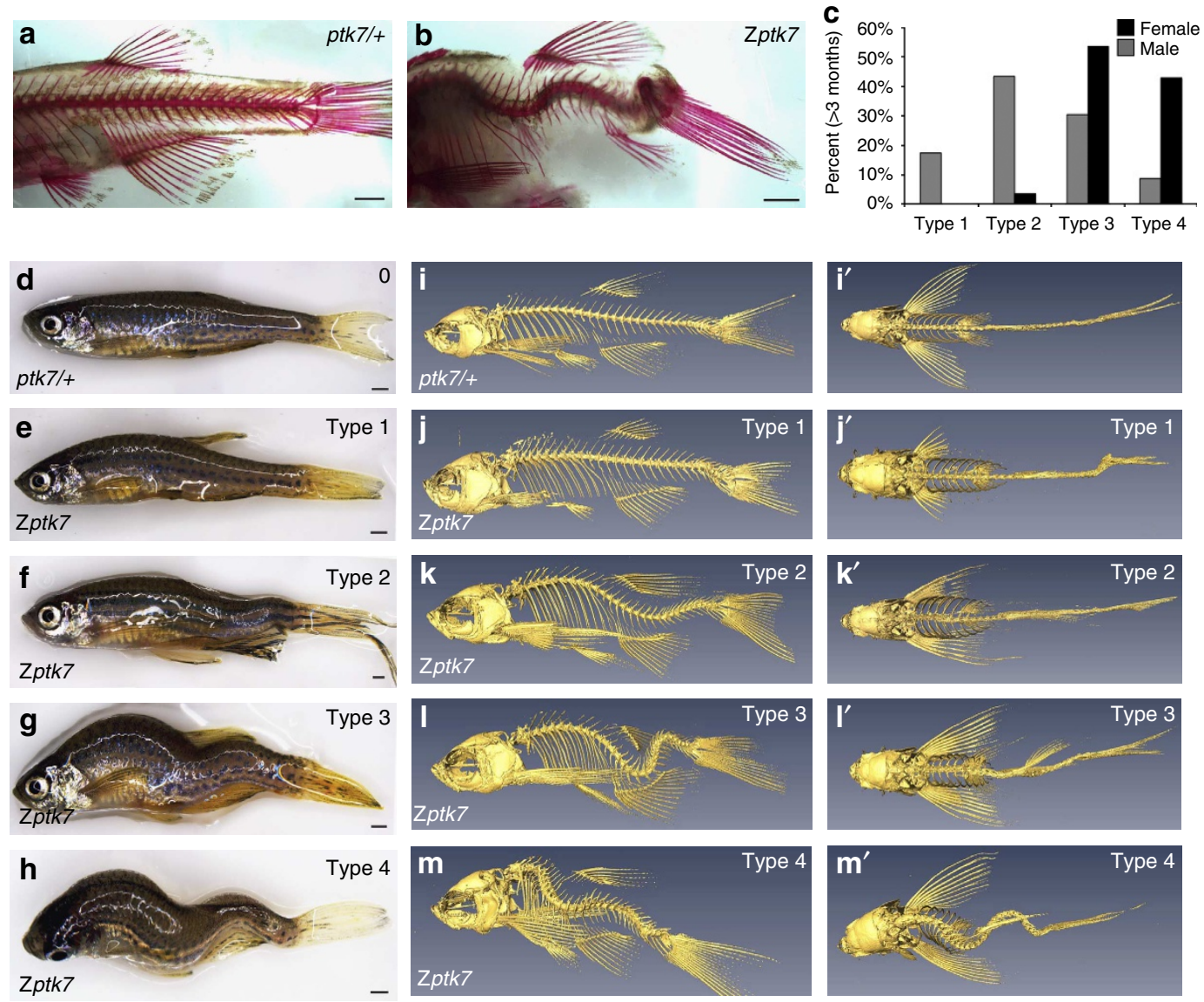

Figure 1 | Zygotic ptk7 mutants display late-onset, three-dimensional spinal curvatures. (a,b) Lateral views of trunk and tail skeleton stained with alizarin red for (a) ptk7/ + and (b) zygotic ptk7 (Zptk7) mutant male zebrafish at 6 months of age. Scale bars, 1 mm. (c) Graph depicting phenotypic and gender distributions for type 1-4 classification based on curve severity at sexual maturity ( $\sim 3$ months of age). Quantification is represented as a percentage of the total for male $(n=92)$ and female $(n=58)$ zebrafish. (d-h) Adult ptk7/ + and Zptk7 mutant zebrafish representative of each curve severity class: (d) unaffected ptk7 heterozygote zebrafish (type 0); (e) mild curvature (type 1); (f) moderate curvature (type 2) primarily isolated to the tail; (g) progressed curvature (type 3) with trunk, tail and mild medio-lateral rotations; (h) severe curvature (type 4) with extreme trunk, tail and medio-lateral rotations. Scale bars, $1 \mathrm{~mm}$. (i-m) Lateral and $\left(\mathbf{i}^{\prime}-\mathbf{m}^{\prime}\right)$ dorsal views of three-dimensional microCT renderings of adult (i) ptk7 heterozygote as well as (j) type 1, (k) type 2, (I) type 3 and $(\mathbf{m})$ type 4 Zptk7 mutant zebrafish.

ventral, left and right sides. We calculated dorsal:ventral and left:right length ratios to assess possible length asymmetries in the dorso-ventral and medio-lateral planes, respectively. Asymmetric or 'wedge-shaped' vertebral distortions were associated with the apex of spinal curves in adult fish, consistent with human IS where vertebrae become misshapen with disease progression (Fig. 2g,h) ${ }^{33-36}$. The affected vertebrae occurred throughout the trunk and tail with no strong positional bias (Fig. 2i,j). Analysis of vertebral bone density revealed no significant difference between Zptk7 mutant and ptk7l + animals (Fig. $2 \mathrm{k}-\mathrm{m}$ ).

To pinpoint the onset of scoliosis in mutant animals, the length and physical appearance of $p t k 7 /+$ and Zptk7 mutant larvae were closely monitored over the first 6 weeks of life (Fig. 3a). Curve onset could first be detected in Zptk7 mutants at $8 \mathrm{~mm}$ standard length ( $\sim 28$ days post fertilization $(\mathrm{dpf}))$, as fish began to exhibit mild body distortions ( $n=18 / 23,78 \%$; Fig. 3e) associated with sagittal curvature of the spine (as confirmed by microCT, Fig. $3 \mathrm{f}$ ). Of note, curve development correlated with the onset of a period of accelerated growth (Fig. 3a). By $1.2 \mathrm{~mm}$ standard length $(\sim 40 \mathrm{dpf})$ all Zptk7 mutants displayed obvious axial curvatures, with some showing three-dimensional rotations $(n=5 / 23$; Fig. $3 \mathrm{~m})$ that increased in magnitude up to late juvenile stages. Curve severity did not visibly progress after sexual maturity ( $\sim 3$ months of age) and adult fish could be grouped into four classes (type 1-4) based on increasing magnitude of spinal curvatures (Fig. 1c-m) ${ }^{37}$. Notably, female Zptk7 mutants demonstrated a clear bias towards severe curves (93\%, $n=58$; Fig. 1c). Late-onset spinal curvatures of Zptk7 mutant zebrafish thus closely model the defining attributes of human adolescent IS.

A novel human PTK7 mutation disrupts Wnt signalling activity. To investigate the mutational burden that PTK7 may contribute to human IS, we Sanger sequenced PTK7 coding exons of 96 human IS samples and identified four distinct missense variants (Supplementary Table 1). Two missense variants were rare ( $<1 \%$ frequency in public databases). We genotyped the two variants, single nucleotide polymorphisms (SNPs) rs34021075 and rs34865794 in a follow-up cohort of 755 non-Hispanic white (NHW) cases and 1,392 NHW controls, but neither was significantly associated with IS independently or in combination (Supplementary Table 2).

Of particular interest was a novel heterozygous PTK7 sequence variant identified in a single IS patient and his father (who did not display spinal deformity). The patient, a 15-year-old male of Asian descent, presented with typical features of IS, a 49 degree right thoracic curve and no other associated clinical findings 

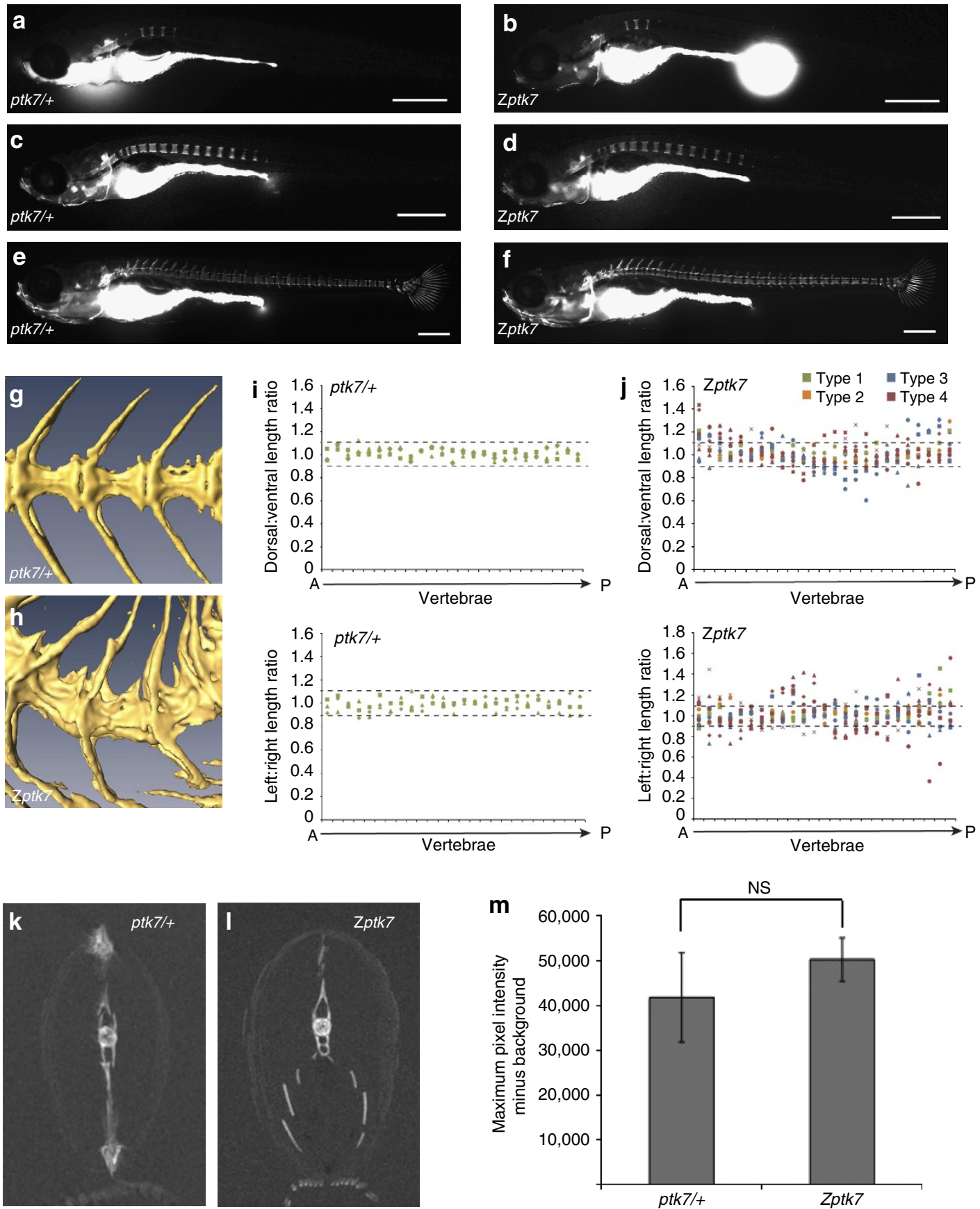

Figure 2 | Zygotic ptk7 mutants do not display vertebral patterning abnormalities but do show vertebral 'wedging' reminiscent of human IS. (a-f) Live calcein staining of $(\mathbf{a}, \mathbf{b}) 4 \mathrm{~mm},(\mathbf{c}, \mathbf{d}) 4.5 \mathrm{~mm}$ and $(\mathbf{e}, \mathbf{f}) 6.0 \mathrm{~mm}(\mathbf{a}, \mathbf{c}, \mathbf{e})$ ptk7 heterozygote and $(\mathbf{b}, \mathbf{d}, \mathbf{f})$ zygotic ptk7 (Zptk7) mutant larvae. All scale bars, $0.5 \mathrm{~mm}$. (g,h) Lateral views of caudal vertebrae from three-dimensional microCT renderings of adult ( $\mathbf{g})$ ptk7 heterozygote and (h) Zptk7 mutant zebrafish. Ptk7 mutant vertebrae display a wedge-shaped morphology at the apex of curves. $(\mathbf{i}, \mathbf{j})$ Graphs depicting dorsal:ventral and left:right length ratios of trunk and tail vertebrae in $p t k 7$ heterozygote $(\mathbf{i} ; n=3)$ and Zptk7 mutant $(\mathbf{j} ; n=12)$ zebrafish. To characterize vertebral shape changes, we measured the length of individual thoracic and tail vertebrae along the dorsal, ventral, left and right sides. We calculated dorsal:ventral and left:right length ratios to assess possible length asymmetries in the dorso-ventral and medio-lateral planes, respectively. Horizontal dotted lines demarcate arbitrary \pm 0.1 , determined by the maximum deviation from 1 in ptk7 heterozygote controls and used to illustrate the deviation of Zptk7 vertebrae from normal. Deviation from $1 \pm 0.1$ is evident at multiple vertebrae in three dimensions along the entire length of the spine. Type 1-4 Zptk7 mutants are grouped by point colour, and each individual fish is represented by a unique point shape and colour. The graph's horizontal axis spans anterior $(A)$ to posterior $(P)$, from the first abdominal vertebrae to the last caudal vertebrae. The Weberian and caudal tail vertebrae were excluded from our analysis. (k,I) Maximum intensity projections (MIP) in the cranio-caudal direction from high-resolution micro-computed tomography (microCT) of (k) ptk7/ + and (I) Zptk7 mutant zebrafish. Dorsal is up and ventral is down. (m) Quantification of maximum pixel intensity corrected for background intensity to infer approximate bone density in $p t k 7 /+(n=3)$ and Zptk7 $(n=12)$ zebrafish. Maximum pixel intensities of the vertebrae were extracted from MIPs made from 10 sequential microCT slices. Three locations from trunk to tail were quantified and averaged for each fish. Error bars represent standard deviation. NS, not significant. 

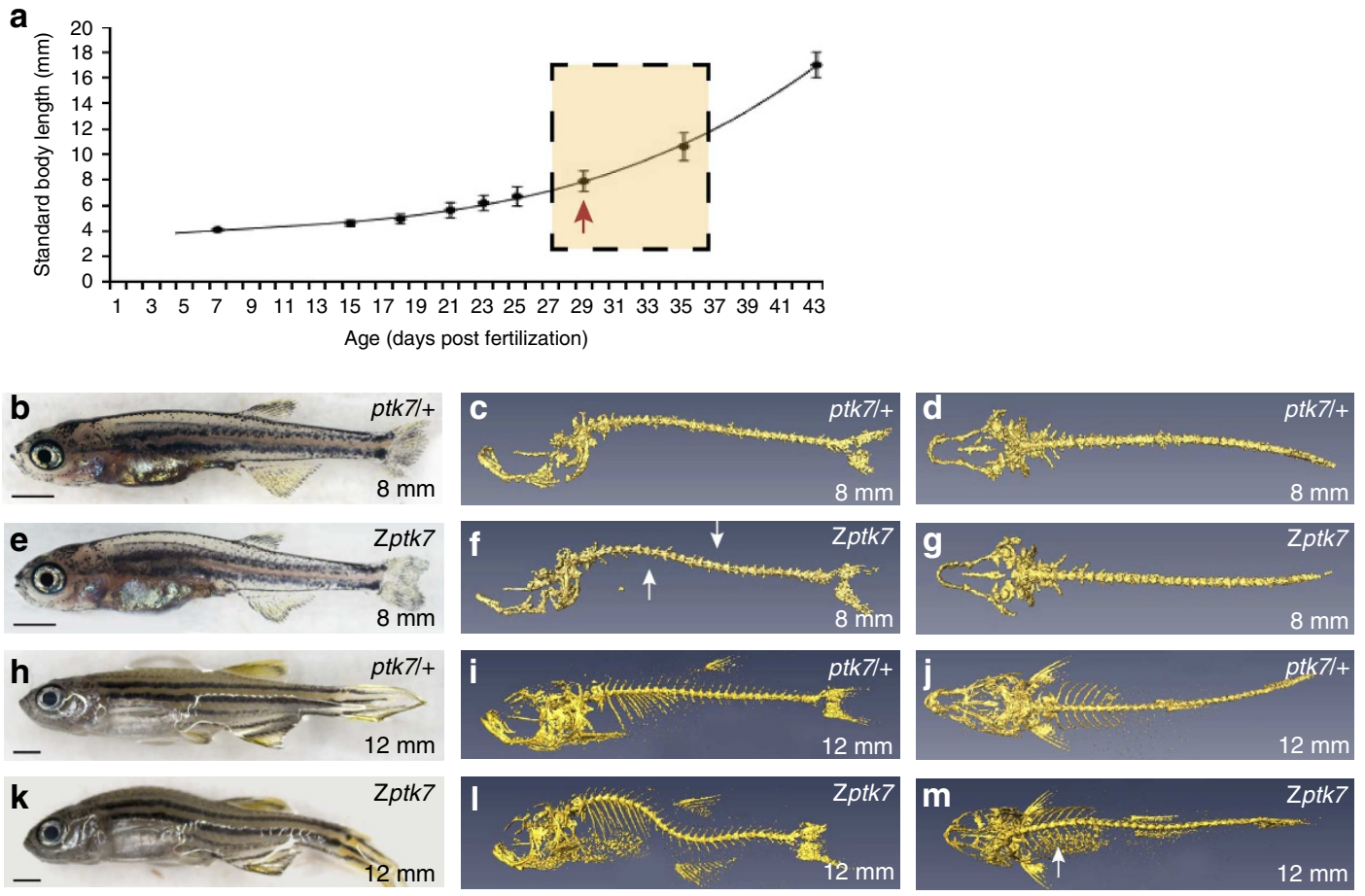

Figure 3 | Zygotic ptk7 mutants develop spinal curvatures that progress rapidly during late larval and early juvenile stages. (a) Graph depicting standard body length measurements ${ }^{65}$ of Zptk7 and ptk7/ + larvae during the first 42 days of development (sample numbers: $6 \mathrm{dpf}=16,14 \mathrm{dpf}=89,17$ $\mathrm{dpf}=72,20 \mathrm{dpf}=51,22 \mathrm{dpf}=48,24 \mathrm{dpf}=47,28 \mathrm{dpf}=48,35 \mathrm{dpf}=48,42 \mathrm{dpf}=40$ ). Zptk7 began to display axial curves at $8 \mathrm{~mm}$ standard body length (red arrow) and curves progress through to juvenile stages, which represents a time of accelerated growth (orange box). After 8 mm standard body length, Zptk7 mutant juveniles were not included as axial curvatures prevented accurate measurements. Data points represent average body length and error bars represent standard deviation. (b-d) ptk7/ + and (e-g) Zptk7 mutant zebrafish at $8 \mathrm{~mm}$ standard body length. (h-j) ptk7/ + and (k-m) Zptk7 mutant zebrafish at $12 \mathrm{~mm}$ body length. Scale bars, $1 \mathrm{~mm}$. (c,f,i, I) Lateral and $(\mathbf{d}, \mathbf{g}, \mathbf{j}, \mathbf{m})$ dorsal views of three-dimensional microCT renderings of $(\mathbf{c}, \mathbf{d}, \mathbf{i}, \mathbf{j})$ ptk7/ + and $(\mathbf{f}, \mathbf{g}, \mathbf{I}, \mathbf{m})$ Zptk7 mutants. At the time of onset, Zptk7 mutants display sagittal spinal curves (arrows in $\mathbf{f}$ ) that can increase in severity to three-dimensional scoliosis (medio-lateral deviation, arrow in $\mathbf{m}$ ).

(Fig. 4a). His curve eventually progressed and required corrective surgery. The PTK7 missense variant occurred in exon 11, predicting a proline to alanine substitution $\left(\mathrm{PTK} 7^{\mathrm{P} 545 \mathrm{~A}}\right)$ within an evolutionarily conserved residue of the sixth extracellular Ig loop. This mutation was novel, as it was not found in any public database (for example, dbSNP or the NHLBI exome variant server), nor in our in-house exome sequencing controls. The variant was also not present in 1,030 additional IS cases and 400 genotyped controls.

We have previously shown that the extracellular domain of zebrafish Ptk7 is essential for its role in Wnt signal transduction $^{24}$. To determine the functional consequence of the PTK7 ${ }^{\mathrm{P} 545 \mathrm{~A}}$ mutation in vivo, we compared the activity of both wild-type (WT) and mutant isoforms following messenger RNA (mRNA) injection into zebrafish embryos. In western blots of whole embryo lysates, PTK7 ${ }^{\mathrm{P} 545 \mathrm{~A}}$ accumulated at greater levels than WT, suggesting increased stability and/or differential modification of PTK $7^{\mathrm{P} 545 \mathrm{~A}}$ protein (Fig. 4b,c; Supplementary Fig. 1). Similarly, membrane localization of yellow fluorescent protein-tagged PTK7 $7^{\mathrm{P} 545 \mathrm{~A}}$ was dramatically increased compared with WT PTK7 (normalized to co-injected membrane-localized monomeric red fluorescent protein, Fig. $4 \mathrm{~d}-\mathrm{i}$ ), suggesting that the P545A variation affects trafficking and/or turnover of PTK7 at the plasma membrane.

Injection of full length zebrafish $p t k 7$ mRNA into one-cell staged MZptk7 mutant embryos can rescue patterning and morphogenesis defects associated with dysregulated Wnt signalling $^{24}$. To determine how the P545A variant affects PTK7 function, we performed similar rescue assays using human
PTK7 mRNA. Strikingly, although injection of $100 \mathrm{pg}$ of WT PTK7 mRNA into one-cell staged embryos significantly rescued PCP-mediated axial extension defects of MZptk7 mutants, injection of equal amounts of PTK7 $7^{\mathrm{P} 45 \mathrm{~A}}$ mRNA had no effect (Fig. 4j-1). This data indicates that the P545A PTK7 mutation disrupts its non-canonical Wnt/PCP signalling activity. Similarly, injection of WT PTK7 mRNA could rescue central nervous system patterning defects caused by wnt8 overexpression (loss of eyes and reduced forebrain at 36 hours post fertilization (hpf)). However, injection of equal amounts of $P T K 7^{P 545 A}$ mRNA failed to rescue, indicating that the $\mathrm{P} 545 \mathrm{~A}$ variant also significantly disrupts PTK7 function in inhibiting canonical Wnt/ $\beta$-catenin activity (Fig. $4 \mathrm{~m}-\mathrm{p}$ ). Together, these data suggest that the P545A variant is a hypomorphic allele of PTK7. Although pathogenesis of the $P T K 7^{\mathrm{P} 545 \mathrm{~A}}$ variant in IS remains uncertain, our results highlight the utility of the zebrafish model for investigating the functional consequence of IS-associated PTK7 mutations in vivo.

MZptk7 mutants develop CVMs. To further investigate the function of Ptk7 in spine morphogenesis, we analyzed vertebrae formation in MZptk7 mutant fish, which demonstrate loss-ofgene function at the earliest stages of embryogenesi ${ }^{24}$. Strikingly, unlike Zptk7 mutants, MZptk7 larvae displayed severe congenital vertebral abnormalities at $4.8 \mathrm{~mm}$ standard length, including missing or smaller vertebral segments, hemivertebrae and/or vertebral fusions (Fig. 5a).

The majority of MZptk7 mutant larvae die before their vertebrae are fully mineralized. Therefore, to determine the role 
a

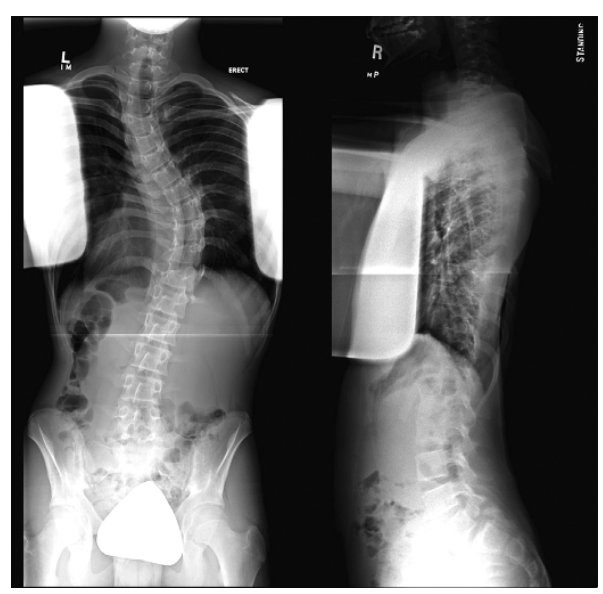

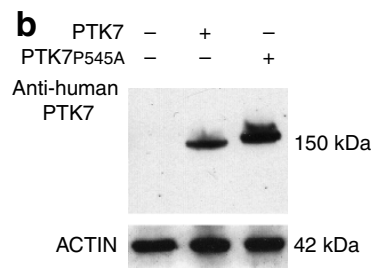

C
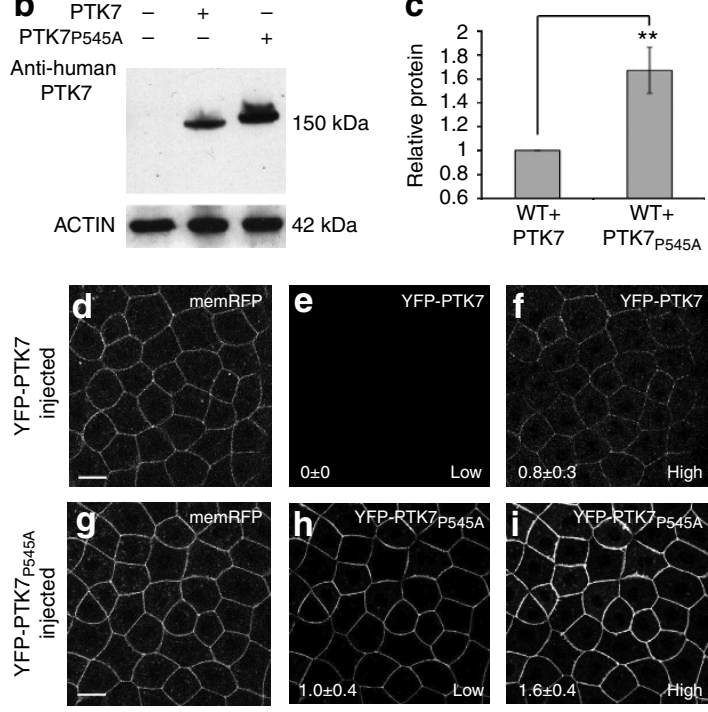
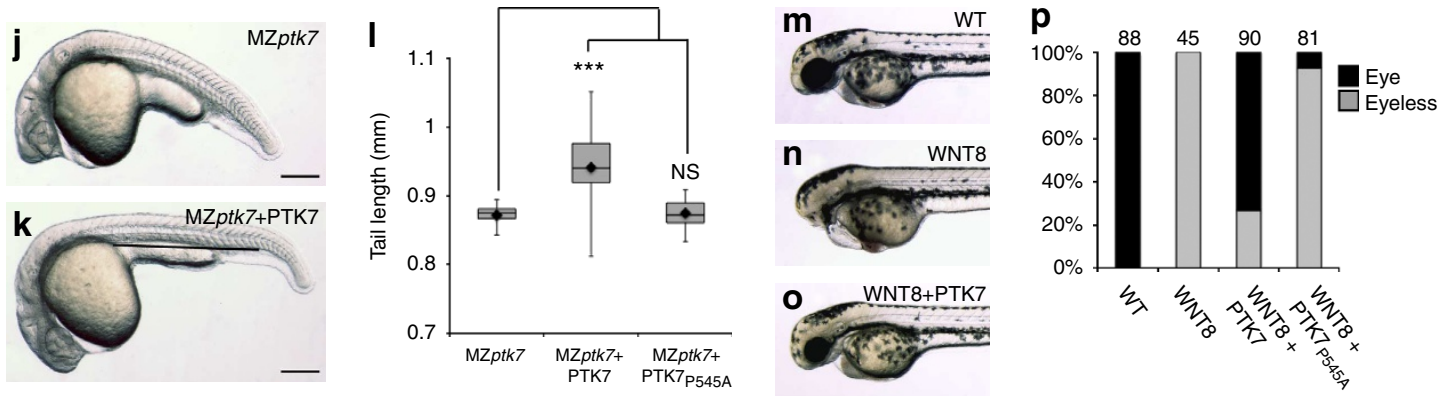

Figure 4 | The P545A variant affects PTK7 Wnt signalling potential. (a) Patient X-ray. (b) Human PTK7 in total cell lysates made from uninjected wildtype (WT) shield-staged zebrafish embryos, or WT embryos injected at the one-cell stage with $250 \mathrm{pg}$ of either PTK7 or PTK $7^{P 545 A} \mathrm{mRNA}$. Actin is used as a loading control. (c) Quantification of relative PTK7 and PTK7 ${ }^{\text {P545A }}$ protein levels $\left(n=3,{ }^{\star \star} P=0.0036\right)$. Protein levels were normalized to actin and PTK7 was set to 1. (d-i) Confocal images of the animal cap of embryos at $60 \%$ epiboly co-injected at the one-cell stage with membrane-localized monomeric red fluorescent protein (membrane RFP) ${ }^{70}$ and either yellow fluorescent protein (YFP)-tagged PTK7 (250 pg) or PTK7 P545A (250 pg) mRNA. Scale bars, $200 \mu \mathrm{m}$. Co-expressed (d) membrane RFP and (e-f) YFP-PTK7 visualized at (e) low (720 V) and (f) high (920 V) intensity. Co-expressed (g) membrane RFP and (h) YFP-PTK7 ${ }^{\text {P545A }}$ at low $(720 \mathrm{~V})$ and $(\mathbf{g})$ high $(920 \mathrm{~V})$ intensity. Numerical values represent average membrane YFP intensity normalized to membrane RFP fluorescent intensity (low $P<0.001$, high $P<0.001 ; n=60$ for each sample). At high (920 V) intensity the YFP-PTK7 ${ }^{\text {P545A }}$ signal was above saturation. Lateral views of (j) MZptk7 and (k) MZptk7 embryos injected with PTK7 (100 pg) mRNA at 24 hpf. Scale bars, $200 \mu \mathrm{m}$. (I) Quantification of tail length $(\mathrm{mm})$ at $24 \mathrm{hpf}$ measured from the base of the yolk extension to the tail tip in MZptk7 mutant embryos $(n=13)$ or MZptk7 mutant embryos injected with $100 \mathrm{pg}$ of either PTK7 $(n=58)$ or PTK7P545A $(n=60)$ mRNA. The bottom and top of each box represent the first and third quartile, respectively; central line represents the median; bars represent the minimum and maximum tail lengths. PTK7 rescues tail extension defects in $M Z p t k 7$ $\left({ }^{\star \star} P<0.0001\right.$, Student's t-test). PTK7 ${ }^{P 545 A}$ mRNA $(100 \mathrm{pg})$ has no effect on axial extension, suggesting reduced PCP signalling activity. (m-p) Overexpression of wnt8 disrupts central nervous system pattern, demonstrated by loss of eyes and reduced forebrain. Lateral views of $48 \mathrm{hpf}$ ( $\mathbf{m}) \mathrm{WT}$ embryos and embryos injected with $(\mathbf{n})$ wnt8 $(10 \mathrm{pg})$ or $(\mathbf{o})$ wnt8 $(10 \mathrm{pg})$ plus PTK7 (250 pg) mRNA. Co-injection of PTK7 (250 pg) mRNA rescues wnt8-induced phenotypes. (p) Quantification of phenotypes observed with injection of wnt8 (10 pg) mRNA, and with co-injection of wnt8 with PTK7 (250 pg) or PTK7 ${ }^{\text {P545A }}$ (250 pg) mRNA. Embryos were scored as being 'eyeless' only if the eye was completely absent. PTK7 $7545 \mathrm{~A}$ is less efficient than PTK7 at rescuing wnt8-induced phenotypes, indicating reduced activity in Wnt/ $\beta$-catenin inhibition. NS, not significant.

for Ptk7 in later aspects of vertebral patterning, we generated genetic chimeras. Briefly, 100-200 MZptk7 mutant cells were transplanted into the ventral domain of shield stage WT embryos (Fig. 5b), which is fated to contribute to multiple lineages of the trunk and tail, including spinal cord and vertebrae ${ }^{38}$. We screened host embryos at $24 \mathrm{hpf}$ for significant MZptk7 cell contribution within the embryonic tail (Fig. 5c), and raised chimeric zebrafish to larval stages. At $6.2-6.5 \mathrm{~mm}$ standard length, calcein staining revealed multiple vertebral malformations in $\sim 27 \%$ of chimeric larvae, including smaller and irregularly shaped vertebral bodies and mislocated sclerotome-derived vertebral spines $(n=8 / 30$, Fig. $5 e)$. These results indicate that chimeric loss of $p t k 7$ in the tail is sufficient to disrupt vertebral patterning at later stages of development. Transplantation of WT cells into a WT embryonic tail bud did not affect vertebral patterning ( $n=42$, Fig. $5 \mathrm{~d}$ ), indicating that observed phenotypes were specific to loss of $p t k 7$.

CVMs are a hallmark of CS, and are thought to arise during early embryogenesis as a result of abnormal segmentation and somite formation ${ }^{3,39}$. To determine whether segmentation is disrupted in MZptk7 mutant embryos, we performed in situ hybridization of her7 and $d l c$ (direct reporters of the Notch segmentation mechanism and nascent somite differentiation) $)^{40}$ within the PSM of 18-20 somite-staged MZptk7 mutant embryos, looking for possible irregularities in gene expression. The PSM domain was broader and expanded relative to total body length in MZptk7 mutants, consistent with a role for Ptk7 in tail-bud patterning and morphogenesis ${ }^{24}$. Of note, although her7 $(n=24)$ 
a
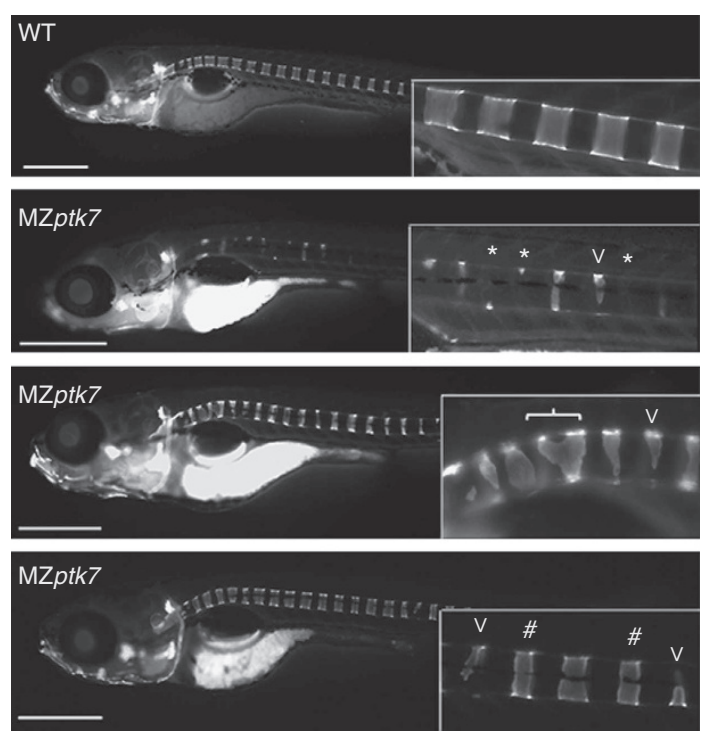

b

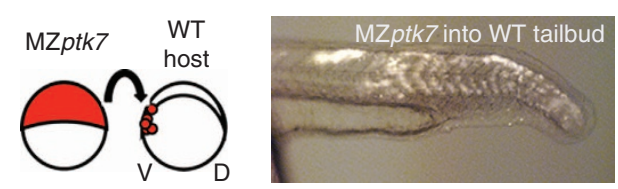

d

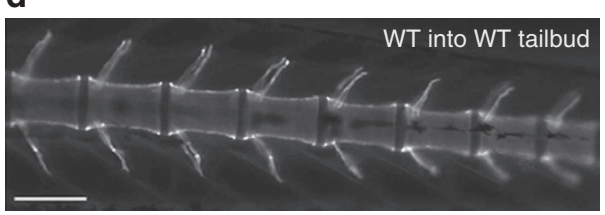

e

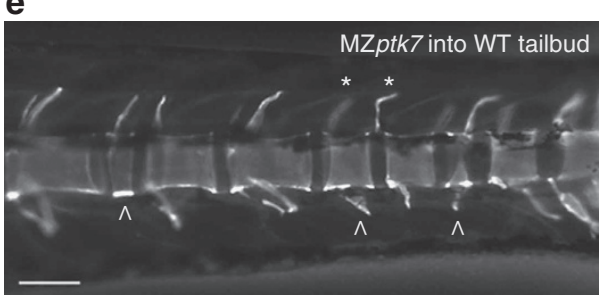

Figure 5 | Maternal-zygotic ptk7 mutant zebrafish display congenital vertebral abnormalities. (a) Live calcein staining of WT and MZptk7 larvae at wildtype $4.8 \mathrm{~mm}$ standard length. Insets highlight representative vertebral malformations present in MZptk7 mutants. Asterisks and arrowheads highlight missing and hemivertebrae, respectively. Bracket highlights vertebral fusions. Hashtags highlight smaller vertebral segments. Scale bars, $0.5 \mathrm{~mm}$.

(b) Transplant strategy used to generate chimeric zebrafish for late larval-stage analysis. MZptk7 cells were labelled with rhodamine red and transplanted at $4 \mathrm{hpf}$ into the ventral marginal zone (fated to become the embryonic tail bud) of shield stage ( $6 \mathrm{hpf}$ ) unlabelled wild-type embryos. ${ }^{38}$ (c) Lateral view of $24 \mathrm{hpf}$ chimeric embryo with fluorescently labelled MZptk7 mutant cells present in the tail of an otherwise wild-type embryo. (d) Lateral views of caudal vertebrae in a control chimeric larvae and (e) in an MZptk7 chimeric larva at 6.2-6.5 mm standard length. MZptk7 cell contribution to the tail leads to vertebral malformations in otherwise wild-type larvae. Arrowheads highlight multiple abnormal vertebrae. Asterisks highlight mislocated vertebral spines (sclerotome derived). Scale bars, $100 \mu \mathrm{m}$.
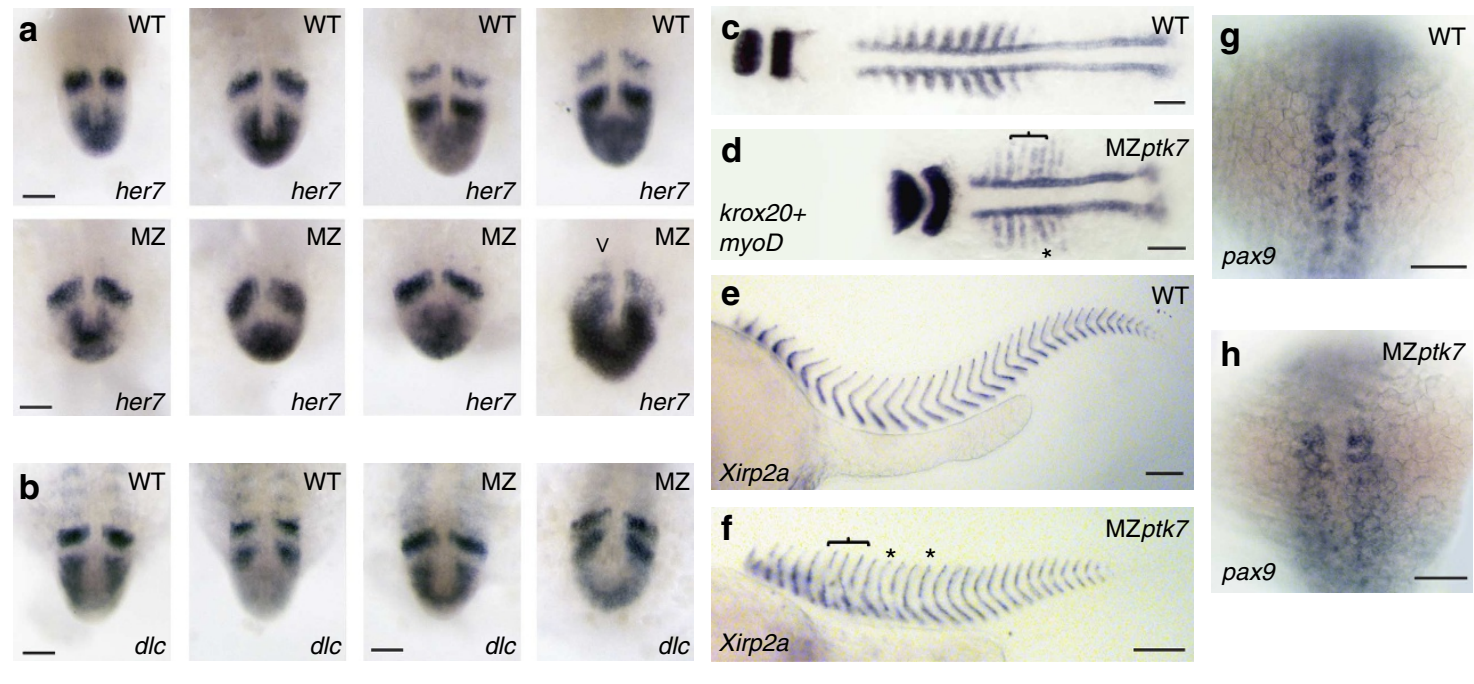

Figure 6 | Maternal-zygotic ptk7 mutant embryos display segmentation defects. (a,b) Whole-mount in situ hybridization (WISH) of somite patterning gene expression at the 18-20 somite stage. All images are posterior views with the embryonic tail bud pointing towards the bottom of the field of view. WT and MZptk7 mutant embryos stained for (a) her7, (b) dlc expression. The expression domains of these markers is expanded within MZptk7 mutants consistent with PSM morphogenetic defects ${ }^{24}$. Arrowhead in a highlights anterior domain asymmetry in one MZptk7 mutant embryo. Scale bars, $50 \mu \mathrm{m}$. (c,d) myoD (somite) staining in (c) WT and (d) MZptk7 embryos to visualize segmentation during somitogenesis. MZptk7 display defects in the regular spacing and morphology of the somites at this stage. Scale bars, $100 \mu \mathrm{m}$. (e,f) Lateral view of WISH for Xirp2a expression to mark somite boarders ${ }^{41}$ in (e) WT and (f) MZptk7 mutant embryos at 40 hpf. The horizontal brackets and asterisks highlight examples of somite fusions. Defects in the shape and size of the somites are also apparent in MZptk7 mutants. Scale bars, $100 \mu \mathrm{m}$. (g,h) pax9 expression in (g) WTand (h) MZptk7 mutant embryos to visualize sclerotome. Scale bars, $100 \mu \mathrm{m}$.

and $d l c(n=18)$ gene expression appeared to oscillate normally in MZptk7 mutant embryos (Fig. 6a,b), occasional irregularities in the symmetry of gene expression were observed ( $n=9 / 24$ in her 7 ;
Fig. 6a), indicating possible defects in somite patterning. Strikingly, myoD and Xirp2a (ref. 41) expression analysis revealed severe defects in the regular morphology and spacing 


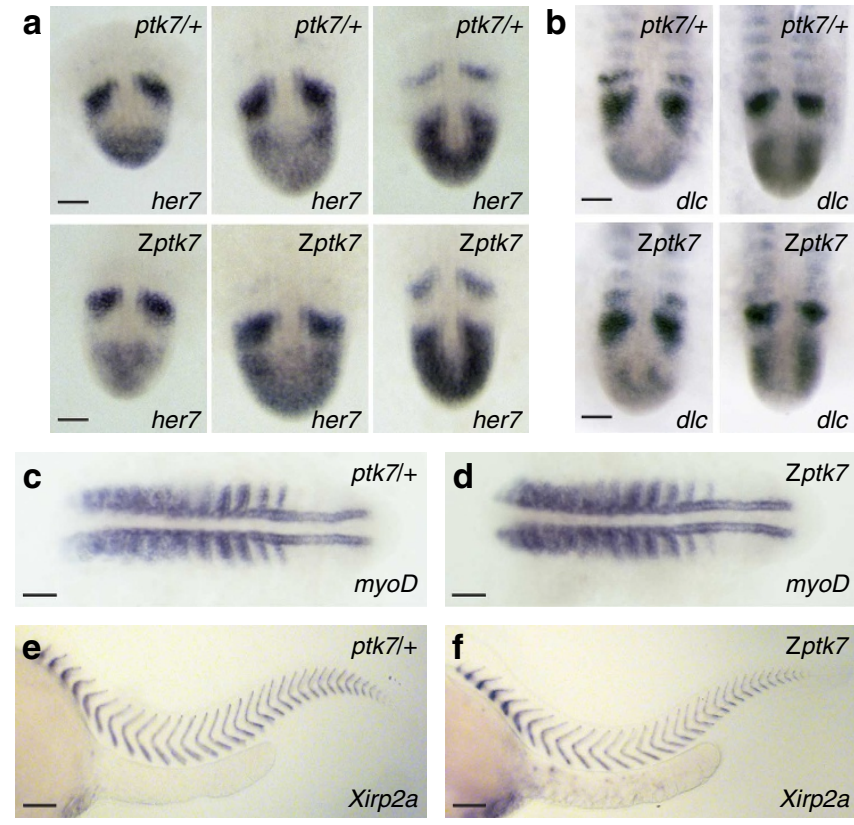

Figure 7 | Zygotic ptk7 mutant embryos do not display segmentation defects. (a) her7 and (b) dlc expression in ptk7/ + and Zptk7 mutant embryos at the 18-20 somite stage. No obvious defects in somitogenesis exist in Zptk7 mutant embryos. Scale bars, $50 \mu \mathrm{m}$. (c,d) myoD (somite) staining in (c) ptk7/ + and (d) Zptk7 embryos to visualize segmentation during somitogenesis. Scale bars, $100 \mu \mathrm{m}$. (e,f) Lateral view of WISH for Xirp2a expression to mark somite boarders ${ }^{41}$ in (e) ptk7/ + and (f) Zptk7 mutant embryos at $40 \mathrm{hpf}$. Zptk7 do not display defects in embryonic segmentation, indicating maternal rescue of the axial patterning defect. Scale bars, $100 \mu \mathrm{m}$.

of the somites in MZptk7 mutants, as well as somite fusions (Fig. 6c-f). Visualization of pax9 gene expression also revealed abnormalities in the regular pattern of sclerotome in MZptk7 mutants (Fig. 6g,h), consistent with abnormal somite patterning.

Importantly, we did not observe segmentation defects in Zptk7 mutant embryos (Fig. 7). This indicates that maternally derived $p t k 7$ gene product (present at the one-cell stage) is sufficient to rescue somitogenesis in Zptk7 mutant zebrafish. More importantly, since maternal $p t k 7$ is not expected to persist beyond early embryonic stages ${ }^{42}$, our data strongly suggest that CVMs observed in MZptk7 mutants are directly linked to early embryonic defects in patterning and morphogenesis.

\footnotetext{
Abnormal PCP disrupts segmentation and vertebral patterning. It is well-understood that $\mathrm{Wnt} / \beta$-catenin signalling within the PSM is required for axial segmentation ${ }^{43-45}$. Since MZptk7 display activated $\mathrm{Wnt} / \beta$-catenin signalling in the embryonic tailbud $^{24}$, we treated WT embryos with the canonical Wnt $/ \beta$ catenin agonists $\mathrm{LiCl}^{46}$ or 6 -bromoindirubin- $3^{\prime}$-oxime $(\mathrm{BIO})^{47}$ throughout somitogenesis to determine the consequence on segmentation and vertebral patterning. Both regiments resulted in somite boundary defects at $40 \mathrm{hpf}$ (Fig. 8a,b) as well as smaller and/or fused vertebral segments at larval stages (Fig. 8c,d), which closely phenocopied MZptk7 mutant animals.

Ptk7 is also required for non-canonical Wnt/PCP signalling. Although previous studies suggest that somite segmentation is unaffected in PCP mutant zebrafish ${ }^{48}$, our analysis of $40 \mathrm{hpf}$ vangl2 mutant embryos (also known as trilobite, deficient for a core and specific regulator of PCP signal transduction $)^{49}$ revealed segmentation defects that were similar to MZptk7 animals $(n=8 / 8$, Fig. $8 \mathrm{e})$. Furthermore, injection of $X d d 1$ mRNA
}
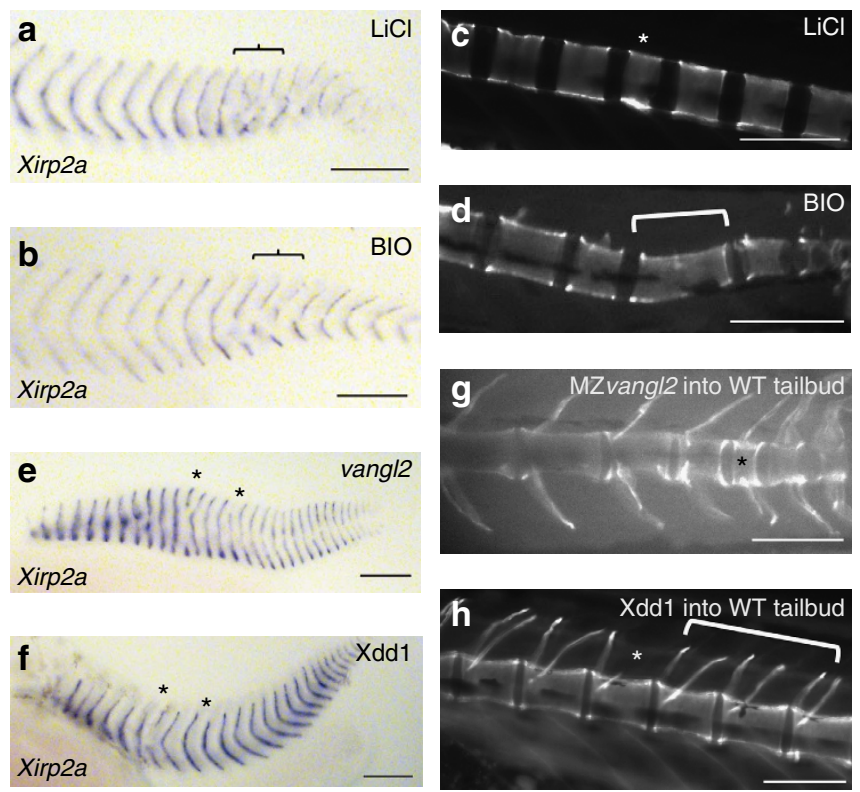

Figure 8 | Abnormal non-canonical Wnt/PCP or canonical Wnt/ק-catenin signalling lead to defects in embryonic segmentation and to subsequent vertebral abnormalities. $(\mathbf{a}, \mathbf{b})$ Lateral view of whole-mount in situ

hybridization (WISH) at $40 \mathrm{hpf}$ for Xirp2a expression to mark somite boundaries ${ }^{41}$ in embryos treated during mid-somitogenesis (10 somites$24 \mathrm{hpf}$ ) with canonical Wnt/ $\beta$-catenin agonist (a) $0.1 \mathrm{M} \mathrm{LiCl}$ or (b) $10 \mu \mathrm{M}$ BIO. Brackets highlight segmental fusions. Scale bars, $100 \mu \mathrm{m}$. (c,d) Lateral views of live calcein staining in 5-6 mm standard length wild-type larvae treated throughout somitogenesis (bud stage-24 hpf) with (c) $10 \mu \mathrm{M} \mathrm{LiCl}$ or (d) $1 \mu \mathrm{M} \mathrm{BIO}$. Scale bars, $100 \mu \mathrm{m}$. Activation of canonical Wnt/ $\beta$-catenin signalling leads to vertebral abnormalities phenotypically similar to MZptk7 loss-of-function including smaller vertebral segments (white asterisk) and/or vertebral fusions (white bracket). (e,f) Lateral view of WISH for Xirp2a expression to mark somite boundaries ${ }^{41}$ in (e) vangl2 mutant embryos and (f) embryos injected with Xdd1 (150 pg) mRNA. Asterisks highlight examples of somite fusions. Scale bars, $100 \mu \mathrm{m}$. Disruption of non-canonical Wnt/PCP signalling results in defects in embryonic segmentation. $(\mathbf{g}, \mathbf{h})$ Lateral views of live calcein staining in wild-type host larvae at $6.2-6.5 \mathrm{~mm}$ standard length that were transplanted with (g) MZvang/2 mutant cells or (h) cells injected with Xdd1 (150 pg) mRNA Transplantation was performed to bypass early lethality of MZvang/2 mutants or Xdd1 overexpression. Donor cells were transplanted into the ventral margin (future tail bud) of wild-type hosts. ${ }^{38}$ Scale bars, $100 \mu \mathrm{m}$. Disruption of non-canonical Wnt/PCP signalling in the tail-bud region results in vertebral abnormalities phenotypically similar to MZptk7 loss-offunction, including smaller vertebral segments (black asterisk), asymmetric (white bracket) or mislocated vertebral arches (white asterisk).

(a dominant-negative Dishevelled construct that specifically disrupts PCP $)^{50}$ produced similar phenotypes ( $n=15 / 18$; Fig. $8 f$ ).

vangl2 mutant and $X d d 1$-injected embryos both die before mineralized vertebrae can be visualized. Therefore, to determine the consequence of abnormal PCP on vertebrae formation, we transplanted mutant cells into the tail bud of WT host embryos and scored vertebral development at larval stages. Both MZvangl2 mutant and $X d d 1$-injected chimeras $(n=8 / 12$ and $9 / 16$, respectively) demonstrated malformed vertebral centra and/or vertebral arches analogous to those observed in MZptk7 mutant larvae (Fig. 8g,h).

Therefore, our data indicates that defects in both PCP and Wnt/ $\beta$-catenin signalling can contribute to segmentation defects and vertebral malformations, suggesting novel genetic and developmental etiologies of CS. 


\section{Discussion}

Ptk7 is a key regulator of canonical Wnt/ $\beta$-catenin and noncanonical Wnt/PCP signalling activity and is required for vertebrate embryonic patterning and morphogenesis ${ }^{24,26,28}$. However, maternal rescue of early developmental abnormalities in Zptk7 mutant zebrafish reveals an important later requirement in spine morphogenesis. As demonstrated, Zptk7 mutant zebrafish develop fully penetrant, late-onset, three-dimensional curvatures of the spine at late larval/early juvenile stages-a period associated with accelerated growth. Females demonstrate a strong bias for severe curves, which can progress in magnitude until sexual maturity. Zptk7 mutant phenotypes thus closely model defining attributes of human adolescent IS. As stated, while IS has been difficult to model in more conventional lab animals, spinal curvature is a common phenotype among fish ${ }^{23,51}$. It has been postulated that cranial-to-caudal spinal loads generated by teleost locomotion mimic biomechanical forces and spinal loads resulting from human upright posture-a contributing factor in IS development and progression. Indeed a number of heritable late-onset scoliosis models have been reported in fish ${ }^{37,52}$, although genetic lesions responsible for these phenotypes have not yet been identified. Zptk7 mutant zebrafish thus represent the first genetically defined developmental model of human IS.

A comprehensive investigation into the contribution of PTK7 mutations in human IS is yet to be performed. However, conservation of Ptk7 function between zebrafish and humans allowed us to directly assess the consequence of a novel PTK7 sequence variant identified in a single IS patient. Remarkably, the $P T K 7^{\mathrm{P} 545 \mathrm{~A}}$ allele disrupts both canonical Wnt/ $\beta$-catenin and non-canonical Wnt/PCP signalling activity, consistent with a role for dysregulated Wnt signalling in IS. Ptk7 is known to interact with Wnt ligands, Frizzled 7 and LRP6 co-receptors ${ }^{28,53,54}$, suggesting that PTK7 is a functional component of the Wnt receptor complex. Since receptor complex endocytosis is an essential component of both Wnt/PCP and Wnt/ $\beta$-catenin signal activation ${ }^{55-57}$, observed increases in $\mathrm{PTK}^{\mathrm{P} 545 \mathrm{~A}}$ plasma membrane localization suggest that the P545A variant could disrupt Wnt signalling by altering complex trafficking. Pathogenesis of the $P T K 7^{\mathrm{P} 545 \mathrm{~A}}$ variant in IS remains to be determined. Despite strong conservation of the PTK7 P545 residue among mammals, it is not conserved in zebrafish confounding requisite 'knock-in' studies. Nevertheless, our functional and genetic results suggest that PTK7 and other Wnt signalling pathway members warrant further re-sequencing in large IS cohorts.

Unexpectedly, maternal-zygotic $p t k 7$ mutant larvae develop vertebral malformations typically associated with CS. Although canonical $\mathrm{Wnt} / \beta$-catenin signalling activity has previously been implicated in segmentation ${ }^{3,44}$, we demonstrate that noncanonical Wnt/PCP defects may also contribute to vertebral malformations and CS pathogenesis. Previous studies have correlated PCP-related axial defects (neural tube disorders) with $\mathrm{CVMs}^{58,59}$. Our analysis of Vangl2 mutant embryos and chimeras clearly show a direct role for PCP in segmentation and vertebral patterning. Of interest, MZptk7 mutants did not exhibit severe misregulation or asymmetries in somite gene patterning that are typically observed following disruption of core segmentation genes ${ }^{60-62}$. However, mild abnormalities/ asymmetries in PSM domain morphology may be sufficient to cause the irregular somite defects observed during later stages of development. Consequently, the MZptk7 mutant phenotype may be more reminiscent of non-syndromic mild or moderate cases of CS where individuals display relatively few CVMs at irregular locations along the length of the spine $e^{3}$.

To summarize, depending on the timing of loss of Ptk7 function, MZptk7 and Zptk7 mutant zebrafish develop distinct

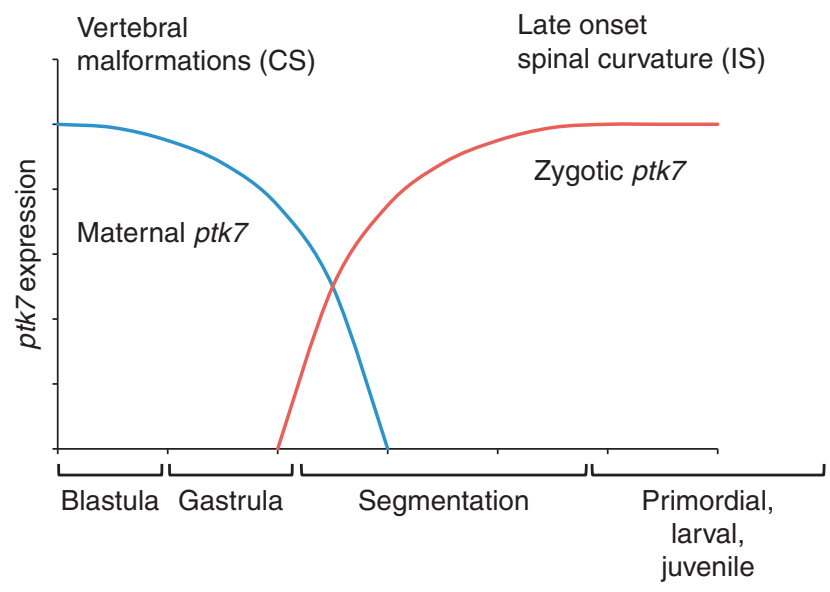

Figure 9 | Ptk7 mutants model CS or IS depending on timing of gene loss-of-function. Maternal $p t k 7$ is deposited in the egg and drives early development including Ptk7-dependent morphogenesis ${ }^{24}$ and segmentation. Loss of maternal (solid blue line) and zygotic ptk7 (solid red line) leads to segmentation defects and vertebral malformations that recapitulate human CS. Zygotic loss of ptk7 leads to late-onset spinal curvature with no underlying vertebral malformations that recapitulate human IS.

spinal deformities that model CS and IS, respectively (Fig. 9). Although CS and IS have long been classified as distinct and separate disorders, a number of independent studies have reported an increased incidence of IS among the family members of CS subjects $5,63,64$. Our study thus provides further evidence that congenital and idiopathic forms of scoliosis may share a common genetic basis.

The biological relationship (if any) between CS and IS is yet to be determined. Although somite and vertebral patterning defects are not observed in Zptk7 mutant embryos, it remains possible that subtle defects in segmentation may be later accentuated by additional genetic or environmental cues. Alternatively, the underlying cause of IS may be completely distinct from CS. Ptk7, Wnt/PCP or Wnt/ $\beta$-catenin signalling have not previously been associated with human IS. However, since the genetics, biomechanics and physiology underlying zebrafish and human development are conserved, shared biological mechanisms underlying spinal curve progression are likely. Zebrafish $p t k 7$ mutant models thus present unique opportunities for investigating the aetiology and pathogenesis of IS and CS, and promise to yield fundamental insights into the developmental, cellular and molecular origins of disease.

\section{Methods}

Zebrafish strains. Established zebrafish husbandry protocols were adhered to and all protocols were performed in accordance with Canadian Council on Animal Care (CCAC) guidelines. MZptk7 and Zptk7 (hsc9) were generated as previously described ${ }^{24}$. The vangl 2 mutant allele tri(tk50f) contains a deletion in the vangl 2 coding sequence ${ }^{49}$. Fish were staged based on standard body length, except in the case of MZptk7 mutant larvae, which were staged based on external anatomy and vital staining 65

Alizarin red. Adult zebrafish were fixed for 1 week in $4 \%$ paraformaldehyde at $4{ }^{\circ} \mathrm{C}$ followed by two, $1 \mathrm{~h}$ washes with phosphate-buffered saline with $0.1 \%$ Tween 20 (PBST). Fish were stained with $0.01 \%$ Alizarin red (Sigma) in $1 \% \mathrm{KOH}$ for 1 week and washed in PBST for 3 days (1 wash/day) Tissues were cleared in $0.5 \%$ trypsin digestion for 1 day followed by multiple washes with PBST. The skin was manually removed and the skeleton was imaged on a Leica MZ16 dissecting scope using AxioVision (Zeiss) software.

Calcein staining. Zebrafish larvae were incubated in 0.2\% Calcein (Sigma), pH 7.5 for $15 \mathrm{~min}$ and washed twice in system water. Larvae were immobilized using 
$0.003 \%$ Tricaine (Sigma) and mounted in 3\% methyl cellulose. Imaging was performed on an Axio Imager.M1 (Zeiss) compound microscope.

MicroCT. Adult zebrafish were fixed in 10\% neutral-buffered formalin for 1 week and mounted in $1 \%$ low-melt agarose (Sigma) in a plastic vial. Specimens were scanned for $1 \mathrm{~h}$ using SkyScan1172 high resolution Micro-CTanner (Bruker micro$\mathrm{CT}$, Belgium) with the X-ray power at $45 \mathrm{kVp}$ and $218 \mu \mathrm{A}$. All three-dimensional Micro-CT data sets were reconstructed with $18 \mu \mathrm{m}$ isotropic resolution. The images were then analyzed using Amira software (TGS Inc., Berlin, Germany)

Plasmids and embryo microinjections. WT human PTK7 was cloned from MGC clone:30347652 using primers: forward $5^{\prime}$-GGGGACAAGTTTGTACAAAAAAG CAGGCTTCGCCACCATGGGAGCTGCGCGGGGATC- $3^{\prime}$ and reverse $5^{\prime}$-GGGA CCACTTTGTACAAGAAAGCTGGGTGTCACGGCTTGCTGTCCACGGTG-3' $P T K 7^{P 545 A}$ was cloned a in two fragments using primers: forward $15^{\prime}$-ATGGGAGCTGCGCGGGGATC-3' ${ }^{\prime}$, reverse $15^{\prime}$-CACTCTGCGAGGCTGCTC- ${ }^{\prime}$, and forward2 $5^{\prime}$-GCAGAGTGGGTGACAGACAA-3', reverse $25^{\prime}$ TCACGGCTTGCT GTCCACGGTG $3^{\prime}$ and assembled by PCR (10 cycles of annealing and extension without primers followed by 30 cycles using WT human PTK7 forward and reverse primers). Both $P T K 7$ and $P T K 7^{P 545 A}$ were inserted into pCS2 + expression vectors (plasmid 22423: pCSDEST, ADDGENE) using Gateway technology (Invitrogen). All mRNA was transcribed using the mMESSAGE mMACHINE System (Ambion) and was injected at the one-cell stage.

PTK7 variant discovery in human IS samples. All research subjects included in the PTK7 re-sequencing study provided written informed consent to participate as approved by the University of Texas Southwestern Medical Center Institutional Review Board. Twenty coding exons of PTK7 were PCR amplified from 96 IS samples as described in the text and sequenced by the Sanger method (oligonucleotide primer sequences and PCR conditions available on request). This identified four nonsynonymous changes. Two highly conserved nonsynonymous SNPs, SNPs rs34021075 and rs34865794, were genotyped by Taqman assay in 736 IS NHW cases and $740 \mathrm{NHW}$ controls and tested for allelic association with IS.

Embryonic transplants. MZptk7, MZvangl2 or Xdd1mRNA-injected cells were labelled with rhodamine dextran (red 10,000 MW, Sigma) by injections at the one-cell stage. Labelled cells were transplanted from sphere ( $4 \mathrm{hpf})$ stage embryos into the ventral margin of unlabelled WT hosts at shield stage (6 hpf) as previously described $^{38}$. Embryos were screened at $24 \mathrm{hpf}$ on an Axio Imager.M1 (Zeiss) compound microscope and embryos containing significant rhodamine-labelled cells (mutant/Xdd1 cells) in the embryonic tail were grown to larval stages.

Whole-mount in situ hybridization (WISH). MyoD (ref. 66), krox20 (ref. 67), her7 (ref. 68), and $d l c$ (ref. 40) plasmids were obtained. Ripply1 and Xirp2a were cloned from an embryonic complementary DNA library made from total RNA using oligo $(\mathrm{dT})_{12-18}$ primer (Invitrogen) and SuperscriptII reverse transcriptase (Invitrogen) as per manufacturer's instructions. The following primers were used: ripply 1 forward $5^{\prime}$-GGGACAAGTTTGTACAAAAAGCAGGCTTCAT GAATTCTGTGTGCTTTGCCA-3' , reverse $5^{\prime}$-GGGGACCACTTTGTACAAGA AAGCTGGGTGGTTGAAAGCTGTGAAGTGACT-3', and Xirp2 $a$ forward $5^{\prime}$-GGGGACAAGTTTGTACAAAAAAGCAGGCTTCAGAGAGCATCCAGG CCACTA-3' ${ }^{\prime}$, reverse $5^{\prime}$-GGGGACCACTTTGTACAAGAAAGCTGGGTGGGCC ACACGACTGCAATTTT-3'. PCR products were inserted into pDONR221 vectors (Invitrogen) using Gateway technology (Invitrogen) and linearized using Apal restriction endonuclease. All antisense RNA probes were prepared by in vitro transcription (DIG RNA Labeling Kit, Roche) in the presence of digoxigenin-11-UTP from linearized DNA templates. Embryos were either fixed and flat mounted in $80 \%$ glycerol or cleared in $100 \%$ methanol, mounted in benzylbenzoate:benzylalcohol (2:1) and imaged on an Axio Imager.M1 (Zeiss) compound microscope.

Drug treatment. Embryos were incubated in $10 \mu \mathrm{M} \mathrm{LiCl} \mathrm{(Sigma)} \mathrm{or} 10 \mu \mathrm{M}$ BIO (Sigma) from bud stage ( $10 \mathrm{hpf}$ ) until $24 \mathrm{hpf}$, washed in system water and grown to larval stages for vertebrae analysis. Embryos were incubated in $0.1 \mathrm{M} \mathrm{LiCl}$ or $100 \mu \mathrm{M}$ BIO from late somite stages until $24 \mathrm{hpf}$, washed in system water and fixed in $4 \%$ paraformaldehyde for WISH.

Western blot analysis. Zebrafish embryos were deyolked ${ }^{69}$, followed by lysis in $1 \%$ NP-40 protein lysis buffer. Protein was denatured using $\beta$-mercaptoethanol buffer at $95^{\circ} \mathrm{C}$ for $10 \mathrm{~min}$, and separated on an $8 \%$ SDS-PAGE gel. PTK7 was detected using goat anti-human PTK7 antibody (LSBio) and mouse anti-actin (Sigma) was used as a loading control.

Confocal imaging. Live embryos were immobilized on a coverslip in $1 \%$ agarose, and imaged using a Zeiss LSM 710 microscope. Z-stacks were collected and processed using ImageJ software.

\section{References}

1. Giampietro, P. F. et al. Congenital and idiopathic scoliosis: clinical and genetic aspects. Clin. Med. Res. 1, 125-136 (2003).

2. Goldstein, L. A. \& Waugh, T. R. Classification and terminology of scoliosis. Clin. Orthop. Relat. Res. 93, 10-22 (1973).

3. Giampietro, P. F. Genetic aspects of congenital and idiopathic scoliosis. Scientifica 2012, 152365 (2012).

4. Pourquie, O. Segmentation of the vertebrate spine: from clock to scoliosis. Cell 145, 650-663 (2011).

5. Bulman, M. P. et al. Mutations in the human delta homologue, DLL3, cause axial skeletal defects in spondylocostal dysostosis. Nat. Genet. 24, 438-441 (2000).

6. Sparrow, D. B. et al. Mutations of the LUNITIC FRINGE gene in humans causes spondylocostal dysostosis with a severe vertebral phenotype. Am. J. Hum. Genet. 78, 28-37 (2006).

7. Sparrow, D. B., Guillen-Navarro, E., Fatkin, D. \& Dunwoodie, S. L. Mutations of Hairy-and-Enhancer-of-Split-7 in humans causes spondylocostal dysostosis Hum. Mol. Genet. 17, 3761-3766 (2008).

8. Sparrow, D. B., Sillence, D., Wouters, M. A., Turnpenny, P. D. \& Dunwoodie, S L. Two novel missense mutations HAIRY-AND-ENHANCER-OF-SPLIT-7 in a family with spondylocostal dysostosis. Eur. J. Hum. Genet. 18, 674-679 (2012).

9. Whittock, N. V. et al. Mutated MESP2 causes spondylocostal dysostosis in humans. Am. J. Hum. Genet. 74, 1249-1254 (2004).

10. Janicki, J. A. \& Alman, B. Scoliosis: review of diagnosis and treatment. Paediatr. Child Health 12, 771-776 (2007).

11. Wise, C. A., Gao, X., Shoemaker, S., Gordon, D. \& Herring, J. A. Understanding genetic factors in idiopathic scoliosis, a complex disease of childhood. Curr. Genomics 9, 51-59 (2008).

12. Gorman, K. F. \& Breden, F. Idiopathic-type scoliosis is not exclusive to bipedalism. Med. Hypotheses 72, 348-352 (2009).

13. Heary, R. F. \& Madhavan, K. Genetics of scoliosis. Neurosurgery 63, 222-227 (2008).

14. Wang, W. J. et al. Top theories for the etiopathogenesis of adolescent idiopathic scoliosis. J. Pediatr. Orthop. 31, S14-S27 (2011).

15. Kou, I. et al. Genetic variants in GPR126 are associated with adolescent idiopathic scoliosis. Nat. Genet. 45, 676-679 (2013).

16. Sharma, S. et al. Genome-wide association studies of adolescent idiopathic scoliosis suggest candidate susceptibility genes. Hum. Mol. Genet. 20, 1456-1466 (2011).

17. Takahashi, Y. et al. A genome-wide association study identifies common variants near LBX1 associated with adolescent idiopathic scoliosis. Nat. Genet. 43, 1237-1240 (2011).

18. Janssen, M. M., de Wilde, R. F., Kouwenhoven, J. W. \& Castelein, R. M. Experimental animal models in scoliosis reasearch: a review of the literature. Spine J. 11, 347-358 (2011).

19. Ouellet, J. \& Odent, T. Animal models for scoliosis research: state of the art, current concepts and future perspective applications. Eur. Spine J. 22, 81-95 (2013).

20. Akel, I. et al. The effect of calmodulin antagonists on scoliosis: bipedal C57BL/6 mice model. Eur. Spine J. 18, 499-505 (2009).

21. Oyama, J., Murai, I., Kanazawa, K. \& Machida, M. Bipedal ambulation induces experimental scoliosis in C57BL/6J mice with reduced plasma and pineal melatonin levels. J. Pineal Res. 40, 219-224 (2006).

22. Fagan, A. B., Kennaway, D. J. \& Oakley, A. P. Pinealectomy in the chicken: a good model of scoliosis? Eur. Spine J. 18, 1154-1159 (2009).

23. Gorman, K. F. \& Breden, F. Teleosts as models for human vertebral stability and deformity. Comp. Biochem. Physiol. C Toxicol. Pharmacol. 145, 28-38 (2007).

24. Hayes, M., Naito, M., Daulat, A., Angers, S. \& Ciruna, B. Ptk7 promotes non-canonical Wnt/PCP-mediated morphogenesis and inhibits Wnt/ $\beta$ catenin-dependent fate decisions during vertebrate development. Development 140, 1807-1818 (2013)

25. Lee, H. K., Chauhan, S. K., Kay, E. \& Dana, R. Flt-1 regulates vascular endothelial cell migration via a protein tyrosine kinase-7-dependent pathway. Blood 117, 5762-5771 (2011)

26. Lu, X. et al. PTK7/CCK-4 is a novel regulator of planar cell polarity in vertebrates. Nature 430, 93-98 (2004).

27. Paudyal, A. et al. The novel mouse mutant, chuzhoi, has disruption of Ptk7 protein and exhibits defects in neural tube, heart and lung development and abnormal planar cell polarity in the ear. BMC Dev. Biol. 10, 87 (2010).

28. Peradziryi, H. et al. PTK7/Otk interacts with Wnts and inhibits canonical Wnt signalling. EMBO J. 30, 3729-3740 (2011).

29. Puppo, F. et al. Protein tyrosine kinase 7 has a conserved role in canonical Wnt/beta-catenin signalling. EMBO Rep. 12, 43-49 (2011).

30. Wagner, G., Peradziryi, H., Wehner, P. \& Borchers, A. PlexinA1 interacts with PTK7 and is required for neural crest migration. Biochem. Biophys. Res. Commun. 402, 402-407 (2012) 
31. Keller, R. Shaping the vertebrate body plan by polarized embryonic cell movements. Science 298, 1050-1954 (2002).

32. Du, S. J., Frenkel, V., Kindschi, G. \& Zohar, Y. Visalizing normal and defective bone development in zebrafish embryos using the fluorescent chromophore calcein. Dev. Biol. 238, 239-246 (2001).

33. Gorman, K. F., Handrigan, G. R., Jin, G., Wallis, R. \& Breden, F. Structural and micro-anatomical changes in vertebrae associated with idiopathic-type spinal curvature in the curveback guppy model. Scoliosis 5, 10-23 (2010).

34. Lanyon, L. E. \& Rubin, C. T. Static vs dynamic loads as an influence on bone remodelling. J. Biomech. 17, 897-905 (1984).

35. Shea, K. G., Ford, T., Bloebaum, R. D., D’Astous, J. \& King, H. A comparison of the microarchitectural bone adaptations of the concave and convex thoracic spinal facets in idiopathic scoliosis. J. Bone Joint Surg. Am. 86, 1000-1006 (2004).

36. Skedros, J. G., Mason, M. W., Nelson, M. C. \& Bloebaum, R. D. Evidence of structural and material adaptation to specific strains features in cortical bone. Anat. Rec. 246, 47-63 (1996).

37. Gorman, K. F., Tredwell, S. J. \& Breden, F. The mutant guppy syndrome curveback as a model for human heritable spinal curvature. Spine (Phila $\mathrm{Pa}$ 1976) 32, 735-741 (2007).

38. Martin, B. L. \& Kimelman, D. Canonical Wnt signalling dynamically controls multiple stem cell fate decisions during vertebrate body formation. Dev. Cell 22, 223-232 (2012)

39. Kaplan, K. M., Spivak, J. M. \& Bendo, J. A. Embryology of the spine and associated congenital abnormalities. Spine J. 5, 564-576 (2005)

40. Oates, A. C., Mueller, C. \& Ho, R. K. Cooperative function of deltaC and her7 in anterior segment formation. Dev. Biol. 280, 133-149 (2005).

41. Otten, C. et al. Xirp proteins mark injured skeletal muscle in zebrafish. PLoS One 7, e31041 (2012).

42. Giraldez, A. J. et al. Zebrafish MiR-430 promotes deadenylation and clearance of maternal mRNAs. Science 312, 75-79 (2006).

43. Aulehla, A. et al. Wnt3a plays a major role in the segmentation clock controlling somitogenesis. Dev. Cell 4, 395-406 (2003).

44. Aulehla, A. et al. A beta-catenin gradient links the clock and wavefront systems in mouse segmentation. Nat. Cell Biol. 10, 186-193 (2008).

45. Hofmann, M. et al. WNT signalling, in synergy with T/TBX, controls Notch signalling by regulating Dll1 expression in the presomitic mesoderm of mouse embryos. Genes Dev. 18, 2712-2717 (2004).

46. Klein, P. S. \& Melton, D. A. A molecular mechanism for the effect of lithium on development. Proc. Natl Acad. Sci. USA 93, 8455-8459 (1996).

47. Polychronopoulos, P. et al. Structural basis for the synthesis of indirubins as potent and selective inhibitors of glycogen synthase kinase- 3 and cyclindependent kinases. J. Med. Chem. 47, 935-946 (2004).

48. Henry, C. A., Hall, L. A., Hille, M. B., Solnica-Krezel, L. \& Cooper, M. S. Somites in zebrafish doubly mutant for knypek and trilobite form without internal mesenchymal cells or compaction. Curr. Biol. 10, 1063-1066 (2000).

49. Jessen, J. R. et al. Zebrafish trilobite identifies new roles for Strabismus in gastrulation and neuronal movements. Nat. Cell Biol. 4, 610-615 (2002).

50. Sokol, S. Y. Analysis of Dishevelled signalling pathways during Xenopus development. Curr. Biol. 6, 1456-1467 (1996).

51. Gorman, K. F. \& Breden, F. Idiopathic-type scoliosis is not exclusive to bipedalism. Med. Hypotheses 72, 2009-2013 (2009).

52. Takeuchi, K. 'Wavy-fused' mutants in the Medaka, Oryzias latipes. Nature 211, 866-867 (1966)

53. Shnitsar, I. \& Borchers, A. PTK7 recruits dsh to regulate neural crest migration. Development 135, 4015-4024 (2008).

54. Bin-Nun, N. et al. PTK7 modulates Wnt signalling activity via LRP6. Development 141, 410-421 (2014).

55. Chen, W. et al. Dishevelled 2 recruits beta-arrestin 2 to mediate Wnt5A-stimulated endocytosis of Frizzled 4. Science 301, 1391-1394 (2003).

56. Blitzer, J. T. \& Nusse, R. A critical role for endocytosis in Wnt signalling. BMC Cell Biol. 7 (2006).

57. Kikuchi, A., Yamamoto, H., Sato, A. \& Matsumoto, S. New insights into the mechanism of Wnt signalling pathway activation. Int. Rev. Cell Mol. Biol. 291, 21-71 (2011).

58. Allache, R., De Marco, P., Merello, E., Capra, V. \& Kibar, Z. Role of the planar cell polarity gene CELSR1 in neural tube defects and caudal agenesis. Birth Defects Res. A Clin. Mol. Teratol. 94, 176-181 (2012).
59. Kibar, Z. et al. Mutations in VANGL1 associated with neural-tube defects. N. Engl. J. Med. 356, 1432-1437 (2007).

60. Echeverri, K. \& Oates, A. C. Coordination of symmetric cyclic gene expression during somitogenesis by Supressor of Hairless involves regulation of retinoic acid catabolism. Dev. Biol. 301, 388-403 (2007).

61. Holley, S. A., Julich, D., Rauch, G. J., Geisler, R. \& Nusslein-Volhard, C. her1 and the notch pathway function within the oscillator mechanism that regulates zebrafish somitogenesis. Development 129, 1175-1183 (2002).

62. Kawakami, Y., Raya, A., Raya, R., Rodriguez-Esteban, C. \& Belmonte, J. Retinoic acid signalling links left-right asymmetric patterning and bilaterally symmetric somitogenesis in the zebrafish embryo. Nature 435, 165-171 (2005).

63. Maisenbacher, M. K. et al. Molecular analysis of congenital scoliosis: a candidate gene approach. Hum. Genet. 116, 416-419 (2005).

64. Purkiss, S. B., Driscoll, B., Cole, W. G. \& Alman, B. Idiopathic scoliosis in families of children with congenital scoliosis. Clin. Orthop. Relat. Res. 401, 27-31 (2002).

65. Parichy, D. M., Elizondo, M. R., Mills, M. G., Gordon, T. N. \& Engeszer, R. E. Normal table of postembryonic zebrafish development: staging by externally visible anatomy of the living fish. Dev. Dyn. 238, 2975-3015 (2009).

66. Weinberg, E. S. et al. Developmental regulation of zebrafish MyoD in wild-type, no tail and spadetail embryos. Development 122, 271-280 (1996).

67. Oxtoby, E. \& Jowett, T. Cloning of the zebrafish krox-20 gene (krx-20) and its expression during hindbrain development. Nuceic Acids Res. 21, 1087-1095 (1993).

68. Oates, A. C. \& Ho, R. K. Hairy/E(spl)-related (Her) genes are central components of the segmentation oscillator and display redundancy with the Delta/Notch signalling pathway in the formation of anterior segmental boundries in the zebrafish. Development 129, 2929-2946 (2002).

69. Link, V., Shevchenko, A. \& Heisenberg, C. P. Proteomics of early zebrafish embryos. BMC Dev. Biol. 13, 1 (2006).

70. Megason, S. G. \& Fraser, S. E. Digitizing life at the level of the cell: high-performance laser-scanning microscopy and image analysis for in toto imaging of development. Mech. Dev. 120, 1407-1420 (2003).

\section{Acknowledgements}

We gratefully acknowledge Dr Benjamin Alman for helpful discussion, and Angela Morley and Allen Ng for excellent zebrafish care. This work was supported in part by funding from the Canada Research Chairs program, the Canadian Institutes of Health Research (MOP-111075) and the March of Dimes Foundation (\#1-FY13-398) to B.C.; the National Institutes of Health (R01 HD052973), the Crystal Charity Ball and the TSRHC Research Fund to C.A.W.; and a CIHR Doctoral Research Award to M.H.

\section{Author contributions}

M. Hayes and B.C. were involved in the conception, design, zebrafish experimentation and analysis. X.G., C.A.W. were involved in the human sample data collection, analysis and interpretation. L.X.Y., M. Henkelman were involved in the microCT data collection.

\section{Additional information}

Supplementary Information accompanies this paper at http://www.nature.com/ naturecommunications

Competing financial interests: The authors declare no competing financial interests.

Reprints and permission information is available online at http://npg.nature.com/ reprintsandpermissions/

How to cite this article: Hayes, M. et al. ptk7 mutant zebrafish models of congenital and idiopathic scoliosis implicate dysregulated Wnt signalling in disease. Nat. Commun. 5:4777 doi: 10.1038/ncomms5777 (2014).

cc)(1) $\ominus$ This work is licensed under a Creative Commons Attribution (c) ${ }_{\mathrm{BY}} \mathrm{NC}$ NO NonCommercial-NoDerivs 4.0 International License. The images or other third party material in this article are included in the article's Creative Commons license, unless indicated otherwise in the credit line; if the material is not included under the Creative Commons license, users will need to obtain permission from the license holder to reproduce the material. To view a copy of this license, visit http:// creativecommons.org/licenses/by-nc-nd/4.0/ 